\title{
Fratura isolada de parede medial da órbita associada à redução importante de movimentação ocular -Relato de caso
}

\author{
Medial isolated blowout orbital fracture associated with important motility restricition - \\ case report
}

\author{
Rosália M.S. Antunes-Foschini ${ }^{1}$ \\ Harley E. A. Bicas ${ }^{2}$ \\ Antônio Auguusto V.Cruz ${ }^{3}$
}

Trabalho realizado no Hospital das Clínicas da Faculdade de Medicina de Ribeirão Preto da Universidade de São Paulo - USP.

${ }^{1}$ Médica assistente junto ao Setor de Estrabismo e Ortóptica do Hospital das Clínicas da Faculdade de Medicina de Ribeirão Preto.

${ }^{2}$ Professor Titular do Departamento de Oftalmologia e Otorrinolaringologia da Faculdade de Medicina de Ribeirão Preto.

${ }^{3}$ Professor Associado do Departamento de Oftalmologia e Otorrinolaringologia da Faculdade de Medicina de Ribeirão Preto.

Endereço para correspondência: Av. Caramuru, $\mathrm{n}^{\circ}$ 1280, apto. 18 - Ribeirão Preto (SP) CEP 14030-000. E-mail: rantunes@keynet.com.br

Recebido para publicação em 10.10.2001

Aceito para publicação em 13.05.2002

Nota editorial: Pela análise deste trabalho e por sua anuência sobre a divulgação desta nota, agradecemos aos Drs. Maurício Brik e Roberto Abucham.

\section{RESUMO}

Homem de 25 anos apresentou diplopia em todas as posições do olhar, associada à movimentação ocular dolorosa, após sofrer traumatismo contuso em região orbitária direita. Tomografia computadorizada revelou fratura isolada indireta de parede medial de órbita direita. A correção cirúrgica da fratura levou ao desaparecimento da queixa de diplopia e melhorou o campo de visão binocular.

Descritores: Fraturas orbitárias/cirurgia; Traumatismos oculares; Diplopia/etiologia; Estrabismo; Movimentos oculares; Visão binocular; Campos visuais/fisiologia; Relato de caso

\section{INTRODUÇ̃̃̃O}

As fraturas da parede orbitária podem ser classificadas em duas categorias: as que acometem e as que não acometem o rebordo orbitário. Aquelas que não acometem o rebordo orbitário também são chamadas de internas. São causadas por traumatismos na região orbitária. As teorias que tentam explicar o mecanismo ${ }^{(1)}$ dessa fratura são duas: o aumento repentino da pressão orbitária é transmitido à parede, que, por ser mais frágil, rompe-se; a segunda diz respeito à transmissão da força exercida pelo trauma através das estruturas ósseas, rompendo-se a parede no local mais frágil. As paredes medial e inferior, por suas reduzidas espessuras, são as mais freqüentemente afetadas, mas a fratura de parede medial raramente produz distúrbios funcionais ${ }^{(2-3)}$ daí sendo relacionada a uma baixa proporção de diagnósticos corretos ${ }^{(3)}$.

A fratura da parede medial da órbita, quando isolada, não é comumente diagnosticada por exames radiográficos e dificilmente causa complicações $^{(4-5)}$. Os sintomas que mais incomodam o paciente, e que com freqüência levam ao diagnóstico, são diplopia nas lateroversões, secundária à movimentação ocular restringida, que pode ser dolorosa, enfisema orbital acentuado e enoftalmia ${ }^{(3-4,6)}$, cujo surgimento pode ser tardio. Esses sintomas e sinais em geral são decorrentes do encarceramento do músculo reto medial e de gordura orbitária no local da fratura, constituindo elementos importantes para indicação de exploração cirúrgica.

Relatamos aqui um caso de fratura isolada de parede medial da órbita, com grandes repercussões sobre a motilidade ocular extrínseca e, portanto, com diplopia em todas as posições do olhar (uma condição rara nessas fraturas), mas com bom resultado após a correção da fratura.

\section{RELATO DE CASO}

RCS, 25 anos, pedreiro, natural de Ribeirão Preto, procedente de 
Guatapará. Em conseqüência de queda de uma altura aproximada de dois metros, sofreu traumatismo contuso em região do olho direito (OD), com perda momentânea da consciência. Procurando a Unidade de Emergência do Hospital das Clínicas da Faculdade de Medicina de Ribeirão Preto foi, inicialmente, avaliado pela área de Neurologia, que descartou acometimento neurológico. Foi então encaminhado para avaliação pela área de Oftalmologia, pelo edema e dor na região do OD. Ao exame dessa região, apresentava edema e hematoma palpebral importantes, com dificuldade para abrir o olho. Referia dor ocular, tanto em repouso como à sua movimentação. Sem queixas relativas ao olho esquerdo $(\mathrm{OE})$. Acuidade visual $(\mathrm{AV})$ do $\mathrm{OD}=0,2$ e do $\mathrm{OE}=0,9$. À biomicroscopia, apresentava hemorragia subconjuntival temporal inferior, pupila em midríase fixa e câmara anterior preservada mas com hifema em região lateral inferior. A fundoscopia indireta mostrou palidez de retina em região superior. A tonometria evidenciou OD com $30 \mathrm{mmHg}$ e OE com $14 \mathrm{mmHg}$. Os demais exames do OE revelaram-se normais. Prescritos ciclopentolato $1 \%$ a cada 12 horas, maleato de timolol $0,5 \%$ a cada 12 horas, colírio com sulfato de neomicina, polimixina $\mathrm{B}$ e dexametasona a cada 3 horas e diclofenaco sódico $500 \mathrm{mg}$ de 6 em 6 horas por via oral, para reavaliação após dois dias.

Paciente retornou com $\mathrm{AV} \mathrm{OD}=0,5$ e $\mathrm{OE}=0,9$, com regressão parcial do edema palpebral, referindo embaçamento visual e dor à movimentação do OD em todas as direções do olhar, sobretudo na elevação e abdução. À biomicroscopia apresentava edema corneal leve e pequena quantidade de células em câmara anterior, com tonometria de $32 \mathrm{~mm} \mathrm{Hg}$. A fundoscopia normalizou-se. Não foi avaliada, neste retorno, a posição ocular. Adicionada acetazolamida $250 \mathrm{mg}$ por via oral de $12 \mathrm{em} 12$ horas.

No quarto dia após o acidente, com a regressão do edema palpebral, paciente passou a referir diplopia em todas as posições do olhar, exceto quando girava a cabeça levemente para a esquerda e para baixo, isto é, com favorecimento do olhar em posição de supradextroversão. $O$ teste de cobertura revelou exotropia e hipertropia do OD em posição primária do olhar (PPO). O teste de motilidade ocular mostrou limitação nas duções em todas as posições, sobretudo em infradução. Dez dias depois a tonometria ainda se conservava alta $(24 \mathrm{~mm} \mathrm{Hg})$ e o OD mantinha as limitações de movimentação e o desvio em PPO. Não havia enoftalmia evidente à inspeção, daí não ter sido realizado exame exoftalmométrico.

A tomografia computadorizada (Figura 1) revelou fratura com afundamento de lâmina papirácea direita e espessamento em músculo reto medial direito (fratura indireta de parede medial da órbita direita). Doze dias após o trauma o paciente apresentava desvio ocular em todas as posições do olhar. (Tabela 1) $\mathrm{O}$ teste das duções passivas revelou limitação da elevação, da abdução e da adução do OD. O campo visual binocular ${ }^{(7)}$ sem diplopia obtido no campímetro de Goldman (Figura 2) mostrou-se contraído.

O paciente foi submetido a cirurgia para correção da fratura orbitária. Após cantólise lateral e eversão da pálpebra inferior, foi feita incisão da conjuntiva do fórnice inferior e conse-

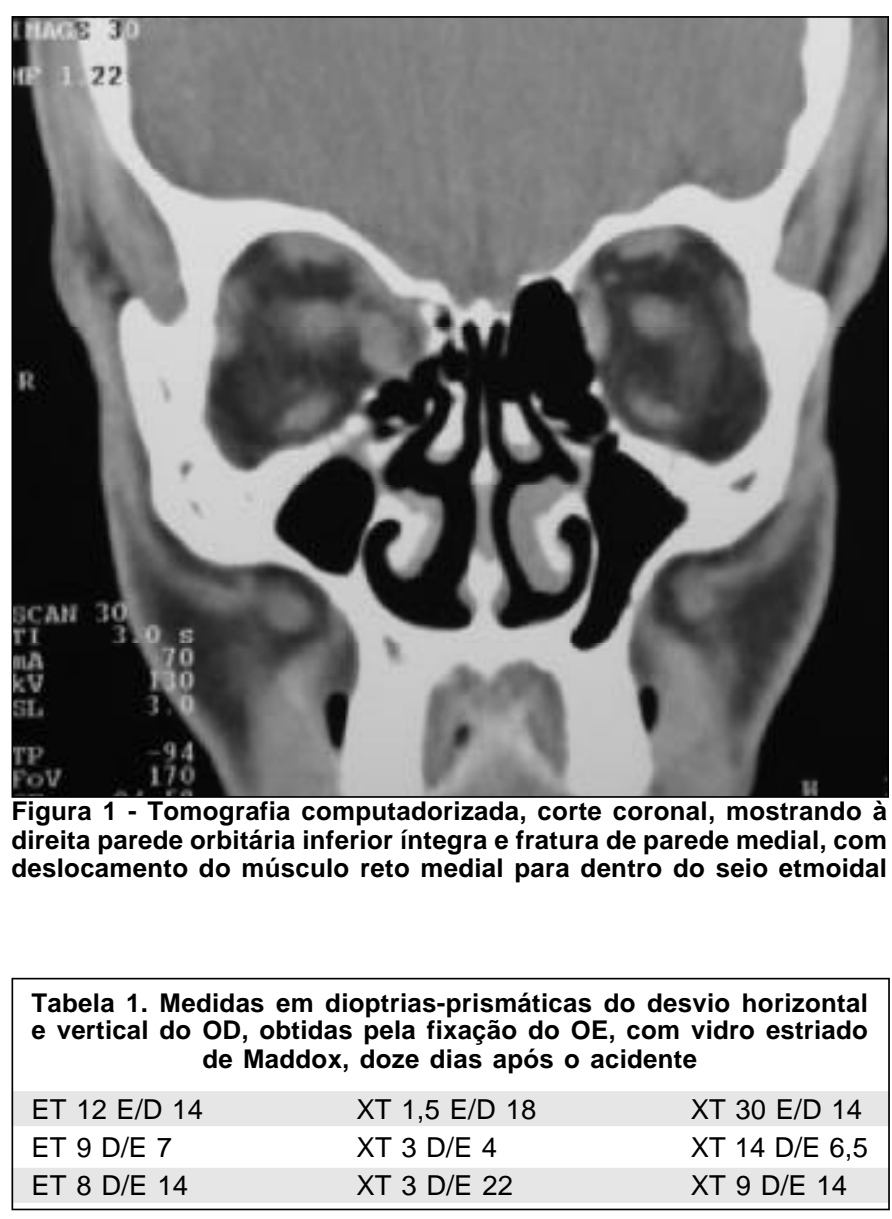

qüente acesso ao rebordo orbitário inferior. $\mathrm{O}$ acesso à região ínfero-medial da órbita foi ampliado por meio de incisão transcaruncular para a parede medial. Após a exposição da lâmina papirácea, toda a fratura medial pôde ser delineada, com a retirada do conteúdo intraorbitário herniado no local da fratura. Colocada placa de polietileno poroso de $0,5 \mathrm{~mm}$ de espessura ao longo da parede medial e feita sutura do periósteo com fio $\mathrm{Vycril} 5,0^{\circledR} \mathrm{e}$ das conjuntivas inferior e transcaruncular com fio Vycril 7, $0^{\circledR}$.

Quinze dias após a cirurgia, a exoftalmometria era de 18 milímetros em ambos os olhos. Vinte dias após a cirurgia, paciente referia melhora da diplopia (Tabela 2), com AV 1,0 em cada um dos olhos.

Cinqüenta dias após a cirurgia, paciente negava diplopia (Tabela 3), com melhora parcial da anisocoria e controle medicamentoso da pressão intra-ocular. Setenta e cinco dias após a cirurgia, paciente negava diplopia, exceto nas posições extremas do olhar. Atualmente encontra-se em seguimento no Setor de Glaucoma, em uso de maleato de timolol $0,5 \%$ a cada 12 horas e tartarato de brimonidina a cada 8 horas, com bom controle da pressão intra-ocular. Ele desenvolveu neste olho hipertensão intra-ocular secundária ao traumatismo (a gonioscopia evidenciou sinéquias e pigmentos em todo o trabeculado). Após sete meses, a exoftalmometria era de 18 milímetros no OD e 17 milímetros no OE. 


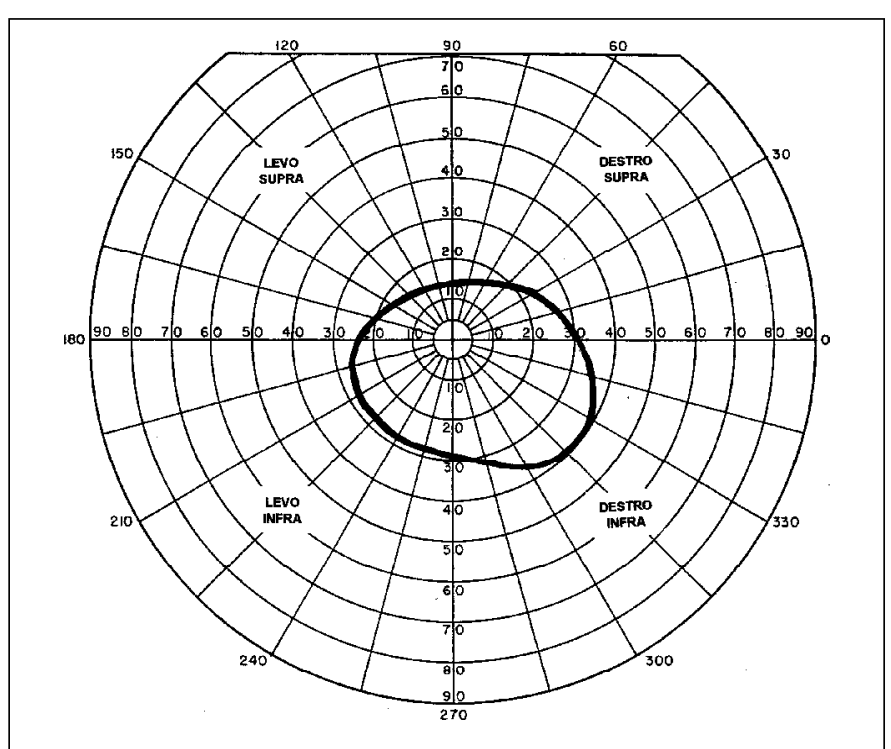

Figura 2 - Campo visual binocular obtido no campímetro de Goldman, doze dias após o acidente

\begin{tabular}{|l|l|}
\hline $\begin{array}{l}\text { Tabela 2. Medidas em dioptrias-prismáticas do desvio horizontal e } \\
\text { vertical do OD, obtidas pela fixação do OE, com vidro estriado de } \\
\text { Maddox, vinte dias após a cirurgia }\end{array}$ \\
\begin{tabular}{lll} 
ET 3 E/D 7 & E/D 5 & XT 12 E/D 7 \\
ET 2 & Zero & XT 4 \\
ET 1 D/E 5 & D/E 3 & XT 6 D/E 7 \\
\hline
\end{tabular}
\end{tabular}

O aumento do campo de visão binocular (campímetro de Goldman) sem diplopia mostra-se na figura 3.

\section{DISCUSSÃO}

As referências à dificuldade para abrir a fenda palpebral (blefaroptose?), diminuição de acuidade visual (cicloplegia?), midríase fixa (paralítica?), com exotropia, desvio vertical e redução de movimentação ocular, poderiam suscitar a hipótese de uma lesão do nervo oculomotor comum, à direita (oftalmoplegia ou oftalmoparesia) relacionada a traumatismo crânio-encefálico, conseqüente à queda. No entanto, a "blefaroptose" tinha, apenas, características mecânicas (edema e hematoma palpebrais), a redução de acuidade visual era explicável por um possível espasmo vascular (a palidez focal e transitória de retina) e por aumento da pressão intra-ocular (com provável edema corneal, aliás depois descrito), enquanto a midríase poderia ser secundária ao próprio trauma direto no segmento anterior do globo ocular. Mais importante, porém, é que o quadro oculomotor tinha pouco em comum com a hipotética paresia de reto medial direito: o exodesvio era muito pequeno em posição primária do olhar $\left(3^{\Delta}\right)$ e apesar de aumentar um pouco em levoversão (adução do OD) chegava a se inverter a esodesvio em dextroversão (abdução do OD), um conjunto de dados mais encontradiço em contenções rotacio-

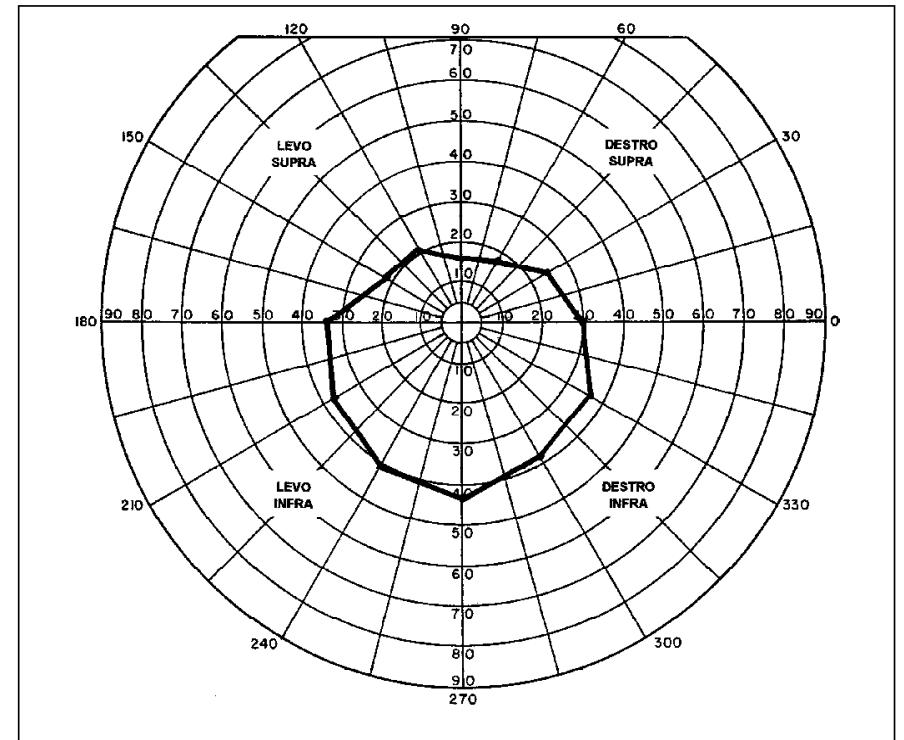

Figura 3 - Campo visual binocular obtido no campímetro de Goldman, cinqüenta dias após a cirurgia

\begin{tabular}{|c|c|c|}
\hline ET 3 E/D 7 & $E / D 6$ & $\mathrm{XT} 10 \mathrm{E} / \mathrm{D} 5$ \\
\hline ET 5 & Zero & XT 5 \\
\hline Zero & D/E 3 & XT 3 \\
\hline
\end{tabular}

nais. Além disso, em oftalmoplegias, predomina o quadro vertical de hipotropia do olho ipsilateral ao nervo afetado, não o contrário (como no caso), que também se invertia entre a supra e a infraversão. A dor, também, conduz o raciocínio a processos orbitários (inflamações, contusões).

A posição em que se relatava ausência de diplopia (em "leve" supradextroversão, isto é, elevação e abdução do OD) está de acordo com a distribuição de desvios (E/D em supraversão, D/E em infraversão; XT em levoversão, ET em dextroversão) e corresponde à situação de repouso anatômico ou "natural" do olho na órbita, o que poderia explicar a condição de desvio causado pela contenção, após a fratura da parede orbitária.

O campo visual binocular mostra uma zona de ausência de diplopia. Isso, obviamente, não quer dizer que haja, também, ausência de desvio mas, tão somente que, nessas posições do campo oculomotor ele esteja compensado (heteroforias).

Após a cirurgia, limitações rotacionais puderam ainda ser evidenciadas, principalmente pelo exame do campo visual binocular (sem diplopia), cuja melhora não foi substancial, embora as medidas do desvio correspondam a valores bem pequenos. Mas é comum que as perturbações oculomotoras associadas a fraturas da parede medial da órbita possam persistir por vários meses após a correção cirúrgica ${ }^{(8)}$.

A elevação da pressão intra-ocular no OD no início e o seu controle com drogas antiglaucomatosas, associada ao resultado da gonioscopia (sinéquias e pigmentos no trabeculado) e 
às medidas normais no OE indicam que a hipertensão intraocular desse olho está diretamente relacionada ao acidente.

\section{ABSTRACT}

A 25-year-old man presented with diplopia in all fields of gaze, associated with painful ductions of the right eye, after suffering a blunt trauma in the region of the right orbit. Computadorized tomography (CT) showed a right medial isolated blowout orbital fracture. Surgical correction eliminated his complaint of diplopia and improved his binocular field of vision.

Keywords: Orbital fractures/surgery; Eye injuries; Diplopia/ etiology; Strabismus; Eye movements; Binocular vision; Visual fields/physiology; Case report

\section{REFERÊNCIAS}

1. Fujino T, Makino K. Entrapment mechanism and ocular injury in orbital blow-out fracture. Plast Reconstr Surg 1980;65:571-6.

2. Kersten RC, Nerad JA. Orbital fractures. In: Tasman W, editor. Duane's clinical ophthalmology. Philadelphia: Lippincott-Raven; 1995. p.2221.

3. Burm JS, Chung CH, Oh SJ. Pure orbital blowout fracture: new concepts and importance of medial orbital wall fracture [commented on Plast Reconstr Surg 1999;104:878-82]. Plast Reconstr Surg 1999;103:1839-49.

4. Segrest DR, Dortzbach RK. Medial orbital wall fractures: complications and management. Ophthal Plast Reconstr Surg 1989;5:75-80.

5. Sanderov B, Viccellio P. Fractures of the medial orbital wall. Ann Emerg Med 1988;17:973-6.

6. DeVisscher JG, van der Wal KG. Medial orbital wall fracture with enophthalmos. J Craniomaxillofac Surg 1988;16:55-9.

7. Feibel RM, Roper-Hall G. Evaluation of the field of binocular single vision in incomitant strabismus. Am J Ophthalmol 1974; 78:800-5.

8. Thaller-Antlanger H. Orbital wall fracture from the ophthalmologic viewpoint. Ther Umsch 1990;47:279-88.

\title{
$5^{\circ}$ Curso de Facoemulsificação $7^{a}$ Jornada de Oftalmologia
}

13 e 14 de Dezembro de 2002
UNIIERSIDADE GAMA FILHO
Rio de Janeiro - RJ

\section{PROMOÇÃO:}

\author{
Serviço de Oftalmologia do Hospital Municipal da Piedade/ \\ Disciplina de Oftalmologia da Escola Médica do Rio de Janeiro \\ Universidade Gama Filho
}

INFORMAÇÕES: Fone: (21) 2595-4097 (Mariângela) 